\title{
Assessment of Right Ventricular Dysplasia
}

Magnetic resonance imaging is currently considered a noninvasive modality of choice for evaluation of patients with suspected right ventricular dysplasia. It has the unique ability to provide tissue characterization in addition to providing functional information. This unit presents the basic techniques for the evaluation of right ventricular dysplasia. The sequence parameters described are most appropriate for the GE 1.5T Signa, and may need to be altered for magnets of different field strengths and manufacturers. The Basic Protocol will take $45 \mathrm{~min}$ to $1 \mathrm{hr}$ to complete. Including the optional sequence 8 to the Basic Protocol will take an additional $\sim 10$ min to perform.

The MR imaging protocol for right ventricular dysplasia is aimed at recognizing two important aspects of the disease: (a) fibro-fatty infiltration of the right ventricle and (b) global and regional right ventricular dysfunction. Hence, the protocol includes (a) black-blood imaging to identify intramyocardial fatty infiltration and (b) bright-blood cine imaging to visualize right ventricular global and regional dysfunction.

For black-blood techniques, breath-hold imaging with double inversion recovery fast-spin echo (FSE) techniques are preferred over traditional spin echo (SE) imaging. These techniques substantially shorten the imaging time and are devoid of respiratory motion artifacts. Black blood inversion in combination with half-Fourier single-shot turbo-spin echo (HASTE) imaging has not been systematically evaluated, but is currently not recommended due to the blurring of detail by this sequence caused by the long echo train length. For bright-blood imaging, steady-state free precession imaging is the most preferred technique (fast imaging employing steady state acquisition-FIESTA, true fast imaging with steady-state-free precession - true FISP, balanced fast field echo-balanced FFE). If those cine sequences are not available, segmented k-space cine gradient echo images (e.g., fast, low-angle shot, FLASH; fast, cardiac-gated gradient echo, FASTCARD) can be used. All images are optimally performed during end expiratory breath-hold.

Table A10.2.1 lists the hardware necessary to perform the procedure, along with the appropriate parameters. The available gradient strength will depend on the scanner and the echo times given in other tables below may be varied accordingly (the smaller the gradient strength, the longer the echo time for a particular scan).

NOTE: Be sure that technologists and nurses have immediate access to any emergency equipment that may be relevant to a given study, or that may be needed for a particular patient, such as crash carts or oxygen.

Table A10.2.1 Equipment Parameters

\begin{tabular}{ll}
\hline Coil type & $\begin{array}{l}\text { Cardiac phased array coil preferred (or } \\
\text { torso phased array coil) }\end{array}$ \\
Gradient coil strength & $25 \mathrm{mT} / \mathrm{m}$ (or whatever the system permits) \\
Cardiac gating & Yes, preferably fiber optic cables \\
Peripheral gating & No \\
Respiratory gating & Yes, to monitor breath hold \\
Respirator & If required by patient \\
Oxygen & If required by patient \\
Motion cushions & NA \\
Use of contrast agents & No \\
\hline
\end{tabular}

Congenital Heart

Disease

Contributed by Harikrishna Tandri and David A. Bluemke

A10.2.1

Current Protocols in Magnetic Resonance Imaging (2003) A10.2.1-A10.2.17

Copyright () 2003 by John Wiley \& Sons, Inc. 


\section{Materials}

Nu-Prep ECG gel

Vermed patches or other non-metallic MR-compatible ECG pad

$50 \mathrm{mg}$ Metoprolol (optional)

\section{Set up patient and equipment}

1. Interview (screen) the patient to ensure that he or she has no contraindications such as cardiac pacemakers or other implants containing ferromagnetic materials that may be problematic for patient safety or good image acquisition. Also be sure to find out if the patient has any health conditions that may require the presence of special emergency equipment during the scanning procedure, or necessitate any other precautions.

Generally, standard screening forms (see APPENDIX 1) are used for all patients scanned in a magnetic resonance system. The MRI centers should allow 20 min before the scheduled MRI exam time for patient preparation.

The presence of any ferromagnetic metals may be a health hazard to the patient when he or she is inside the magnet, and will also affect the image quality. If in doubt as to the exact composition of the items, it is best to exclude patients with any metal implants; see Shellock (2001) for a discussion of what implants may be safely scanned using magnetic resonance.

Patients may be accompanied into the magnet room by a friend or family member who can sit in the room during the scan and comfort the patient as needed. This companion must be screened as well to ensure the absence of loose metal objects on the body or clothing, as well as other items as described above.

2. If the procedure is a research protocol, have the patient sign any necessary consent forms.

3. Have the patient remove any metallic objects, including jewelry, dentures, hearing aids, hairpins, and other objects and secure all items in a safe location. Have the patient change into a gown to eliminate any metal that might be found in clothing.

4. Have the patient wash off any mascara and other makeup to avoid local tissue heating and image artifacts.

5. Inform the patient about what will occur during the procedure, what he or she will experience while in the magnet, and how to behave, including the following:

a. If earplugs, earphones, or headphones are used to protect the ears from the loud sounds produced by the gradients, the patient will be asked to wear one, but will be able to communicate with you at any time during the imaging.

b. The patient may be given a safety squeeze-bulb or similar equipment to request assistance at any time (demonstrate how this works).

c. For good results, the patient should not talk, and should avoid or minimize other movement during each scan-i.e., as long as the banging sounds continue. Between scans, talking is allowed in most cases, but should be avoided when comparative positional studies are being performed; the patient will be informed when this is the case.

d. Nevertheless, the patient may call out at any time if he or she feels it necessary.

e. The procedure will take $\sim 45 \mathrm{~min}$.

f. The MRI technologist should answer any questions that the patient may have.

Assessment of Right Ventricular Dysplasia A10.2.2 
Explain to the patient that a soft pad, which has a receiving coil, will be placed on the chest and that it will aid in creating the images.

The head or feet first position will depend on the manufacturer (feet first is preferred if possible).

7. Practice breath-holds with the patient while preparing for ECG lead placement. Practice a 20 -sec breath-hold with the patient until satisfied that the patient understands the importance of breath-holding at resting lung volume. Ask the patient to take two deep breaths in and out, take another breath in, and hold it.

8. Set up ECG leads as follows:

a. Use an anterior lead placement if possible.

b. Rub the area where electrodes go with Nu-Prep ECG (electrocardiogram) gel.

c. Apply gel to gauze and rub skin gently.

d. Remove excess gel and apply the ECG patches.

9. Place the cardiac phased array coil over the patient's chest just below the clavicle so that the coil lies in the center of the chest and over the patient's heart, which is the region of interest. Secure it with the Velcro or buckle.

10. If needed, place a pillow or other support under the knees to make the patient more comfortable.

11. Align the centering light to the center mark on the anterior coil. Move the patient to the center of the magnet.

Once this step has been performed, so long as the patient does not move on the table, the table itself can be moved and then returned to the same position as before without jeopardizing the positioning of one scan relative to another.

12. If the patient is very anxious, give a mild sedative $30 \mathrm{~min}$ prior to the procedure (this may help reduce the patient's anxiety).

If the patient is known to have frequent ventricular ectopy, the authors recommend the use of $50 \mathrm{mg}$ oral Metoprolol $1 \mathrm{hr}$ prior to the procedure provided that the patient has no contraindications. If ventricular arrhythmias are frequent and will substantially impact image quality, the exam should be terminated at this point.

\section{Sequence 1: Sagittal scout}

13. Set up the scan parameters for sequence 1 as shown in Table A10.2.2. Upon completion of the setup, instruct the patient to take in a deep breath and exhale, take in another deep breath, and hold it. Run sequence 1 according to Table A10.2.2.

\section{Sequence 2: Transverse black blood images}

14. Set up parameters for sequence 2 as shown in Table A10.2.3. Review the sagittal scout images and select an image, which shows the mid left ventricle cavity. Place the localizer line along the orientation of the transverse plane and apply grid lines beginning from the diaphragm to the pulmonary artery.

15. Tell the patient that the next scan involves a series of $\sim 20$-sec breath-holds.

16. Instruct the patient to take in a deep breath and exhale, repeat, take in another deep breath, and hold it. Run sequence 2 according to Table A10.2.3 with the number of slices set to one. 
Table A10.2.2 Primary Clinical Imaging Parameters for Sequence 1:

Sagittal Scout

\begin{tabular}{|c|c|}
\hline Patient position & Supine \\
\hline Scan type & 2-D gradient echo \\
\hline Imaging plane (orientation) & Sagittal \\
\hline Pulse sequence database (PSD) & 2-D fast gradient echo \\
\hline Central slice or volume center & $\begin{array}{l}\text { Laser light centered on mid point } \\
\text { between the sternal notch and the } \\
\text { xiphoid process }\end{array}$ \\
\hline Echo time $\left(T_{\mathrm{E}}\right)$ & Minimum (e.g., $1.5 \mathrm{msec})$ \\
\hline Receiver bandwidth (RBW) & $\pm 31.25 \mathrm{kHz}$ \\
\hline Number of lines per segment & 32 \\
\hline Repeat time $\left(T_{\mathrm{R}}\right)$ & $1 \mathrm{R}$-to-R interval \\
\hline Flip angle (FA) & $15^{\circ}$ \\
\hline Fields of view $\left(\mathrm{FOV}_{x}, \mathrm{FOV}_{y}\right)$ & $400 \mathrm{~mm}, 400 \mathrm{~mm}$ \\
\hline Resolution $(\Delta x, \Delta y)$ & $1.56 \mathrm{~mm}, 2.50 \mathrm{~mm}$ \\
\hline Number of data points collected $\left(N_{\mathrm{x}}, N_{\mathrm{y}}\right)$ & 256,160 \\
\hline Display matrix $\left(D_{\mathrm{x}}, D_{\mathrm{y}}\right)$ & 256,256 \\
\hline Slice thickness $(\Delta z)$ & $10 \mathrm{~mm}$ \\
\hline Number of slices & 4 \\
\hline Slice gap & $8 \mathrm{~mm}$ \\
\hline Number of acquisitions $\left(N_{\mathrm{acq}}\right)$ & 1 \\
\hline Swap read and phase encoding & No \\
\hline Number of cardiac phases & 1 \\
\hline ECG gating & Yes \\
\hline Scan time & 20 heart beats ( 5 heart beats per slice) \\
\hline
\end{tabular}

17. Repeat step 16 until all slices are covered.

Figure A10.2.1 shows the prescription for transverse black blood sequence.

The transverse imaging plane provides the best view of the right ventricular anterior wall and the right ventricular outflow tract. This imaging plane is useful to demonstrate intramyocardial hyperintense $T_{1}$ signals and to evaluate for outflow tract enlargement. Transverse black blood images are acquired at the authors' institution using a double inversion recovery fast-spin echo sequence with blood suppression. If the center has a dedicated cardiac coil, best results are obtained using the anterior coil elements only (posterior coil switched off) to prevent wrap-around artifacts. Also, the authors recommend using an anterior saturation band as shown in Figure A10.2.2. An example of a good quality transverse black blood image sequence is shown in Figure A10.2.3.

\section{Sequence 3: Transverse bright blood cine images}

18. Set up sequence 3 as shown in Table A10.2.4. Prescribe similarly for the transverse black blood images as in sequence 2, i.e., starting from the diaphragm to the pulmonary artery. Inform the patient that a new scan is about to begin.

19. Instruct the patient to take in a deep breath and exhale, ask the patient to repeat, take in another deep breath, and hold it. Run sequence 3 according to Table A10.2.4 with the number of slices set to one slice.

20. Repeat step 19 until the entire region of interest is covered, i.e., all slices are covered.

Assessment of Right Ventricular Dysplasia

Cine imaging in the transverse plane is optimal to assess right ventricular global and regional function visually. One can also assess the right ventricular and atrial chamber enlargement in this plane. As previously mentioned, the most preferred method of cine imaging is using steady-state free precession technique. This allows better endocardial 
Table A10.2.3 Primary Clinical Imaging Parameters for Sequence 2:

Transverse Black Blood Images

\begin{tabular}{|c|c|}
\hline Patient position & Supine \\
\hline Scan type & $\begin{array}{l}\text { Double inversion recovery fast-spin } \\
\text { echo }\end{array}$ \\
\hline Imaging plane (orientation) & Transverse \\
\hline Pulse sequence database (PSD) & FSE-xl \\
\hline Central slice or volume center & Center of heart \\
\hline Echo time $\left(T_{\mathrm{E}}\right)$ & Minimum-full (e.g., 5-10 msec) \\
\hline Receiver bandwidth (RBW) & $\pm 62.5 \mathrm{kHz}$ \\
\hline Echo train length (ETL) & 24 (preferred) to 32 \\
\hline Repeat time $\left(T_{\mathrm{R}}\right)$ & 2 R-to-R intervals \\
\hline Inversion time $\left(T_{\mathrm{I}}\right)$ & $\sim 625 \mathrm{msec}$ \\
\hline Flip angle (FA) & $\mathrm{NA}^{a}$ \\
\hline Fields of view $\left(\mathrm{FOV}_{x}, \mathrm{FOV}_{y}\right)$ & $\begin{array}{l}260 \mathrm{~mm}, 260 \mathrm{~mm} \text { (depends on patient } \\
\text { size and available signal) }\end{array}$ \\
\hline Resolution $(\Delta x, \Delta y)$ & $1.02 \mathrm{~mm}, 1.02 \mathrm{~mm}$ \\
\hline Number of data points collected $\left(N_{\mathrm{x}}, N_{\mathrm{y}}\right)$ & 256,256 \\
\hline Display matrix $\left(D_{\mathrm{x}}, D_{\mathrm{y}}\right)$ & 256,256 \\
\hline Slice thickness $(\Delta z)$ & $5 \mathrm{~mm}$ \\
\hline Number of slices & Variable (usually 8-9 in total) \\
\hline Slice gap & $5 \mathrm{~mm}$ \\
\hline Number of acquisitions $\left(N_{\mathrm{acq}}\right)$ & 1 \\
\hline Swap read and phase encoding & No \\
\hline Slice locations & $\begin{array}{l}\text { From pulmonary bifurcation to dome } \\
\text { of diaphragm }\end{array}$ \\
\hline ZIP 512 & Yes \\
\hline Saturation pulses & $\begin{array}{l}\text { Anterior saturation band over } \\
\text { subcutaneous fat }\end{array}$ \\
\hline Fat suppression & No \\
\hline ECG gating & Yes \\
\hline Scan time & 2-3 min (13-18 sec per slice) \\
\hline
\end{tabular}

${ }^{a}$ The flip angle of the inversion pulse is $180^{\circ}$.

definition when compared to fast gradient echo. For best results while using steady-state free precession in GE scanners, one should manually inspect resonance peaks to insure that the center frequency is water.

An example of good quality image obtained from the transverse bright blood sequence is shown in Figure A10.2.4.

\section{Sequence 4: Vertical long axis scout}

21. Review the transverse black blood images and select an image that shows both the left ventricular cavity and the left atrium, in order to place the localizer in the long axis of the left ventricle parallel to the interventricular septum. Draw the grid line from the left ventricular apex to the mid mitral valve plane, and parallel to the interventricular septum as shown in Figure A10.2.5. Instruct the patient to take in a deep breath and exhale, repeat, take in another deep breath, and hold it. Run sequence 4 according to the parameters shown in Table A10.2.5.

\section{Sequence 5: Four-chamber cine scout}

22. On the diastolic vertical long axis cine image obtained from sequence 4 , place the localizer along the long axis of the left ventricle. Draw the grid line from the left 


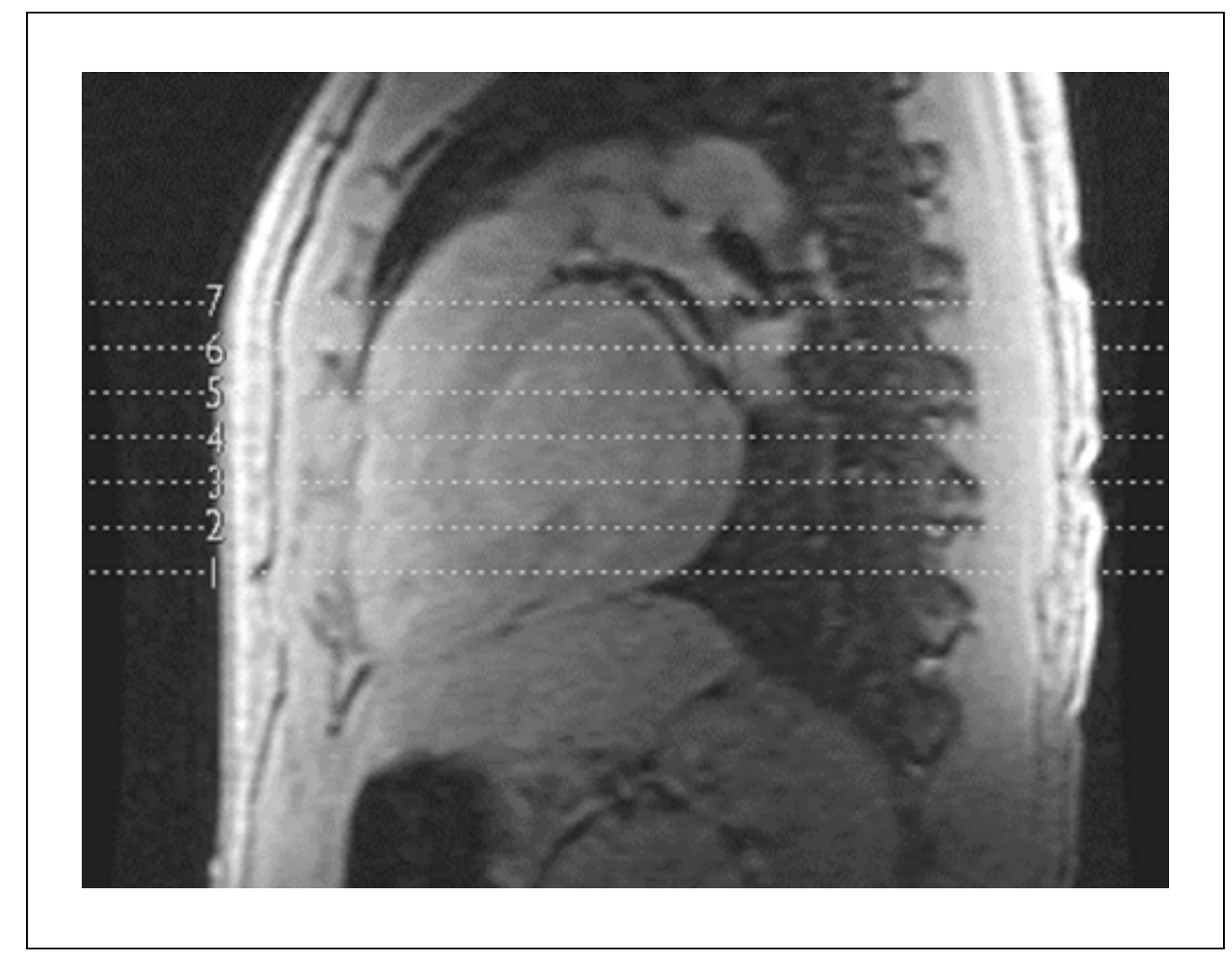

Figure A10.2.1 Prescription of transverse black blood images on sagittal scout image.

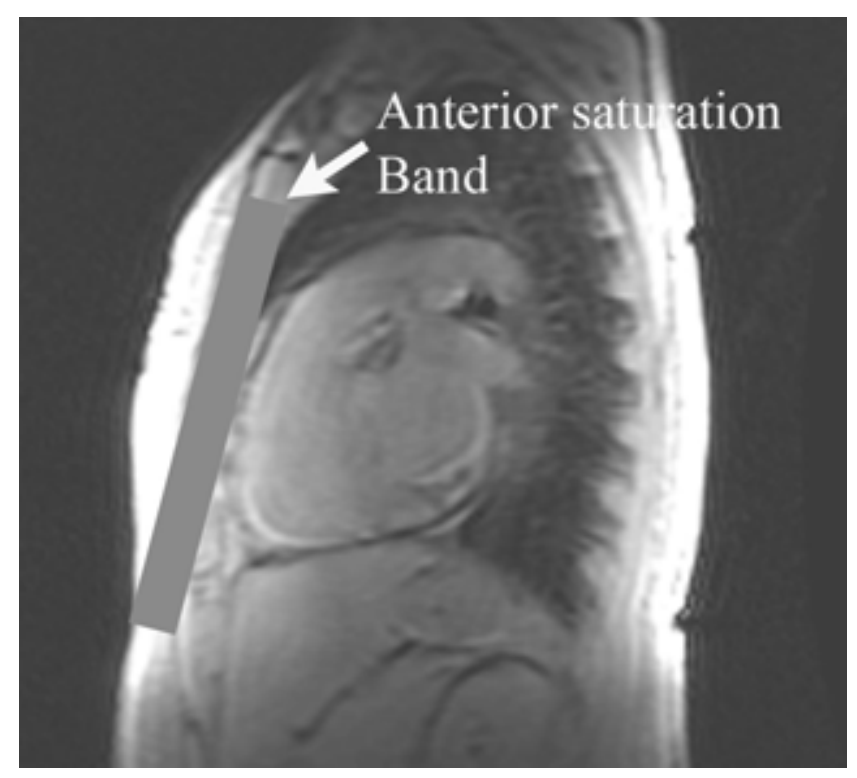

Figure A10.2.2 Sagittal scout image showing the positioning of an anterior saturation band for the transverse black blood images.

Assessment of Right Ventricular Dysplasia

A10.2.6 


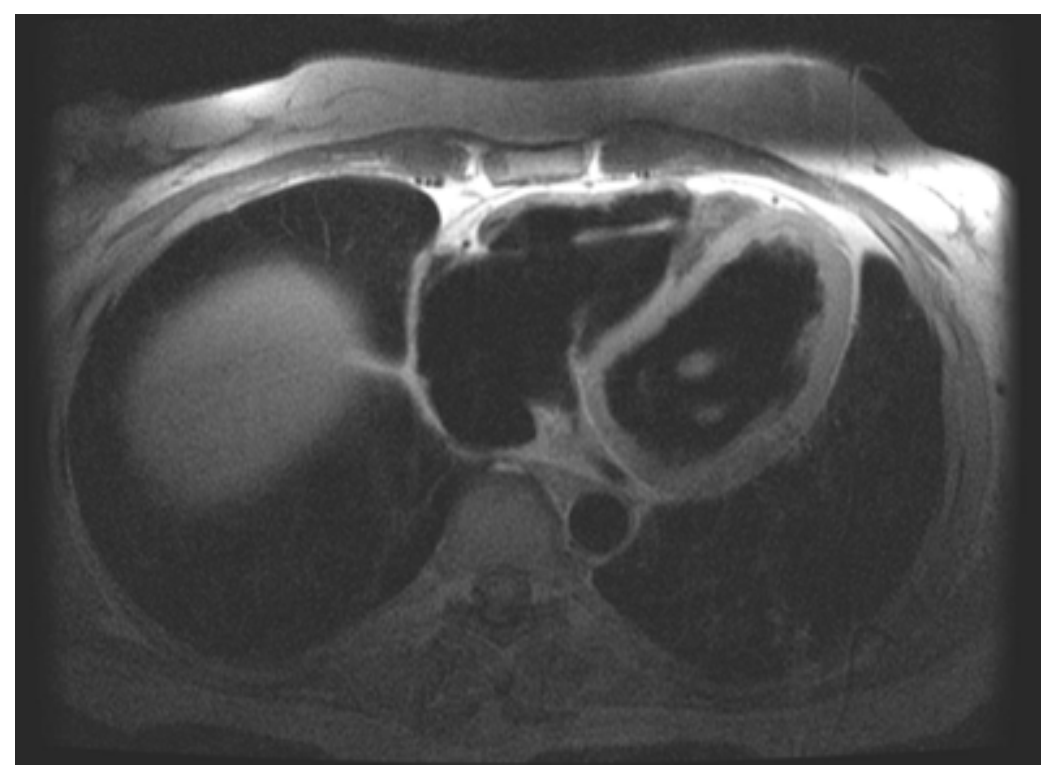

Figure A10.2.3 An example of a good quality transverse black blood sequence image. Note the use of anterior coil elements only and the use of an anterior saturation band

Table A10.2.4 Primary Clinical Imaging Parameters for Sequence 3: Transverse Bright Blood Cine Images

\begin{tabular}{|c|c|}
\hline Patient position & Supine \\
\hline Scan type & Cardiac cine gradient echo \\
\hline Imaging plane (orientation) & Transverse \\
\hline Pulse sequence database (PSD) & 2-D FIESTA/true-FISP/balanced FFE \\
\hline Central slice or volume center & Center of heart \\
\hline Echo time $\left(T_{\mathrm{E}}\right)$ & Minimum (e.g., $1.3 \mathrm{msec}$ ) \\
\hline Receiver bandwidth (RBW) & $\pm 125 \mathrm{kHz}$ \\
\hline Number of lines per segment & $12-16$ \\
\hline Repeat time $\left(T_{\mathrm{R}}\right)$ & 30-40 msec (temporal resolution) \\
\hline Delay time $\left(T_{\mathrm{D}}\right)$ & Minimum (i.e, $10 \mathrm{msec}$ ) \\
\hline Flip angle (FA) & $45^{\circ}$ \\
\hline Fields of view $\left(\mathrm{FOV}_{x}, \mathrm{FOV}_{y}\right)$ & $360 \mathrm{~mm}, 360 \mathrm{~mm}$ \\
\hline Resolution $(\Delta x, \Delta y)$ & $1.41 \mathrm{~mm}, 2.25 \mathrm{~mm}$ \\
\hline Number of data points collected $\left(N_{\mathrm{x}}, N_{\mathrm{y}}\right)$ & 256,160 \\
\hline Display matrix $\left(D_{\mathrm{x}}, D_{\mathrm{y}}\right)$ & 256,256 \\
\hline Slice thickness $(\Delta z)$ & $8 \mathrm{~mm}$ \\
\hline Number of slices & Variable (usually 5-7 in total) \\
\hline Slice gap & $4 \mathrm{~mm}$ \\
\hline Number of acqusitions $\left(N_{\mathrm{acq}}\right)$ & 1 \\
\hline Swap read and phase encoding & No \\
\hline Slice locations & $\begin{array}{l}\text { From pulmonary bifurcation to dome } \\
\text { of diaphragm }\end{array}$ \\
\hline Saturation pulses & None \\
\hline Fat suppression & No \\
\hline Number of cardiac phases & 20-30 (heart rate dependent) \\
\hline ECG gating & Yes \\
\hline Scan time & $\begin{array}{l}\sim 1 \min (10-14 \mathrm{R}-\text { to- } \mathrm{R} \text { intervals per } \\
\text { slice) }\end{array}$ \\
\hline
\end{tabular}




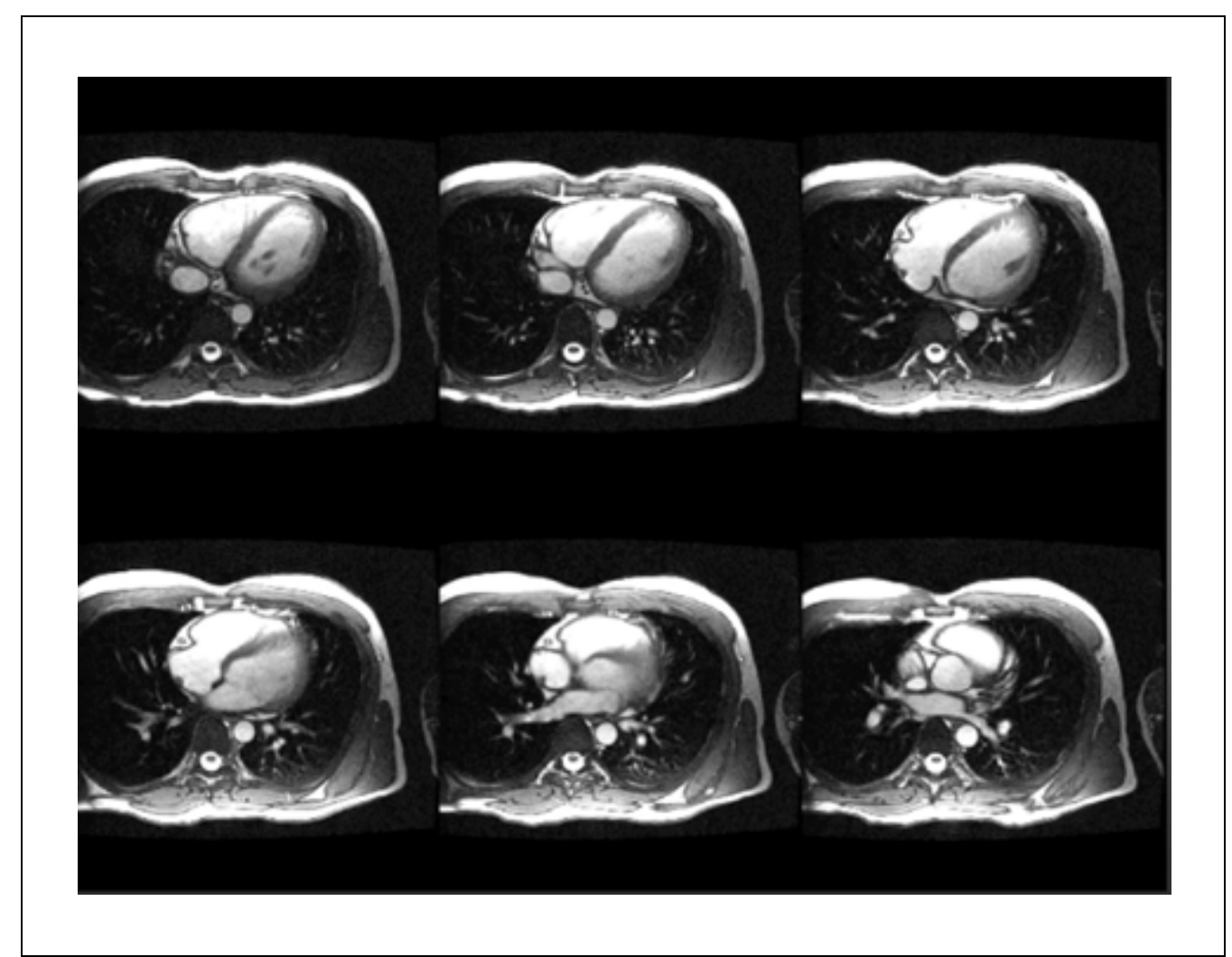

Figure A10.2.4 An example of a transverse bright blood cine sequence image.

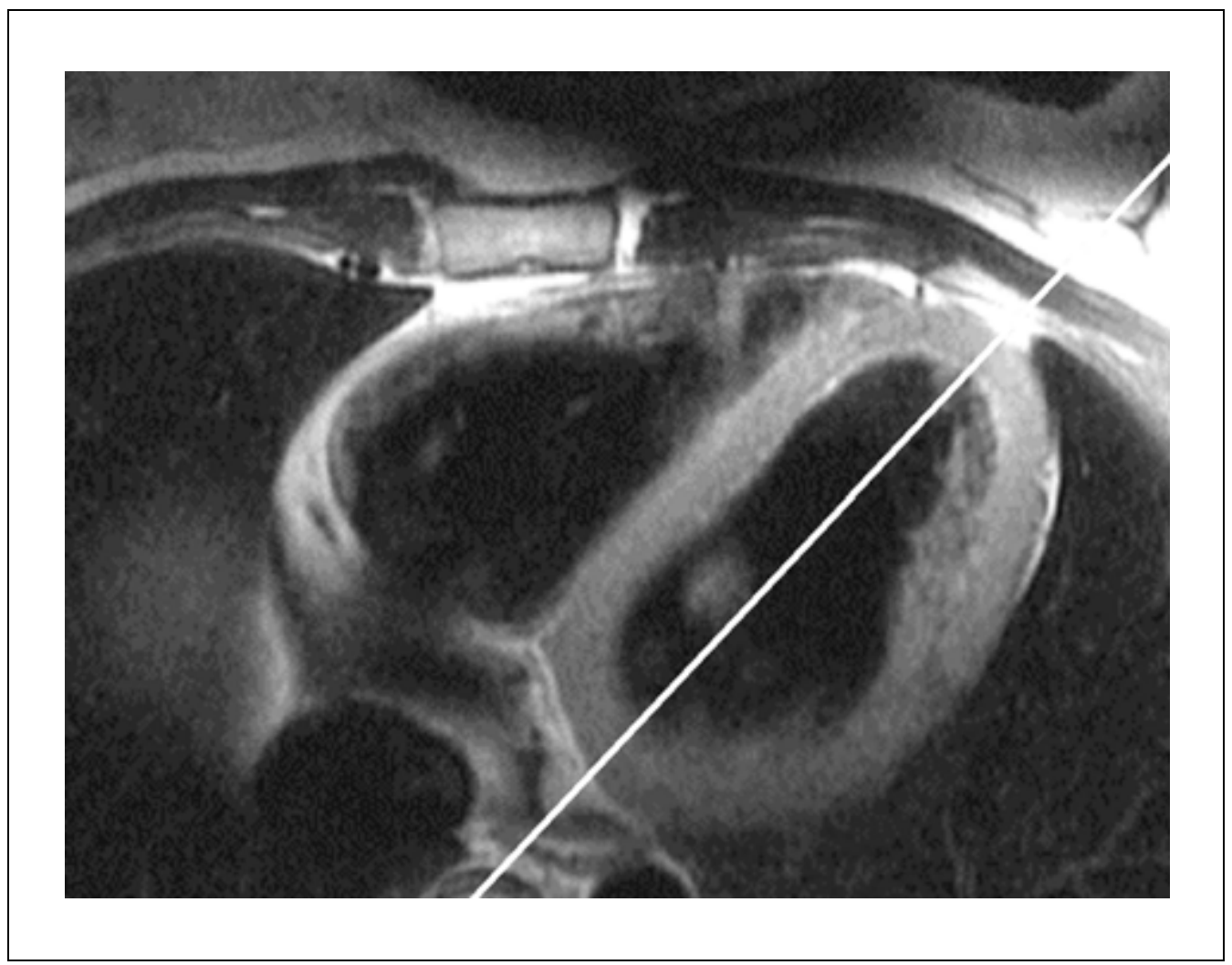

Figure A10.2.5 Prescription of a vertical long axis view of the left ventricle from a transverse image.

Assessment of Right Ventricular Dysplasia

A10.2.8 
Table A10.2.5 Primary Clinical Imaging Parameters for Sequence 4: Vertical Long Axis Scout

Patient position

Scan type

Imaging plane (orientation)

Pulse sequence database (PSD)

Central slice or volume center

Echo time $\left(T_{\mathrm{E}}\right)$

Receiver bandwidth (RBW)

Number of lines per segment

Repeat time $\left(T_{\mathrm{R}}\right)$

Delay time $\left(T_{\mathrm{D}}\right)$

Flip angle (FA)

Fields of view $\left(\mathrm{FOV}_{x}, \mathrm{FOV}_{y}\right)$

Resolution $(\Delta x, \Delta y)$

Number of data points collected $\left(N_{\mathrm{x}}, N_{\mathrm{y}}\right)$

Display matrix $\left(D_{\mathrm{x}}, D_{\mathrm{y}}\right)$

Slice thickness $(\Delta z)$

Number of slices

Slice gap

Number of acquisitions $\left(N_{\text {acq }}\right)$

Swap read and phase encoding

Number of cardiac phases

ECG gating

Scan time
Supine

Gradient echo

Oblique sagittal

Fast gradient echo

Center of left ventricle

Minimum (e.g., $1.3 \mathrm{msec}$ )

$\pm 32 \mathrm{kHz}$

32

Minimum ( 128-320 msec, temporal resolution)

Minimum (e.g., $10 \mathrm{msec})$

$15^{\circ}$

$360 \mathrm{~mm}, 360 \mathrm{~mm}$

$1.41 \mathrm{~mm}, 2.81 \mathrm{~mm}$

256, 128

256, 256

$8 \mathrm{~mm}$

1

NA

1

No

1

Yes

4 heart beats

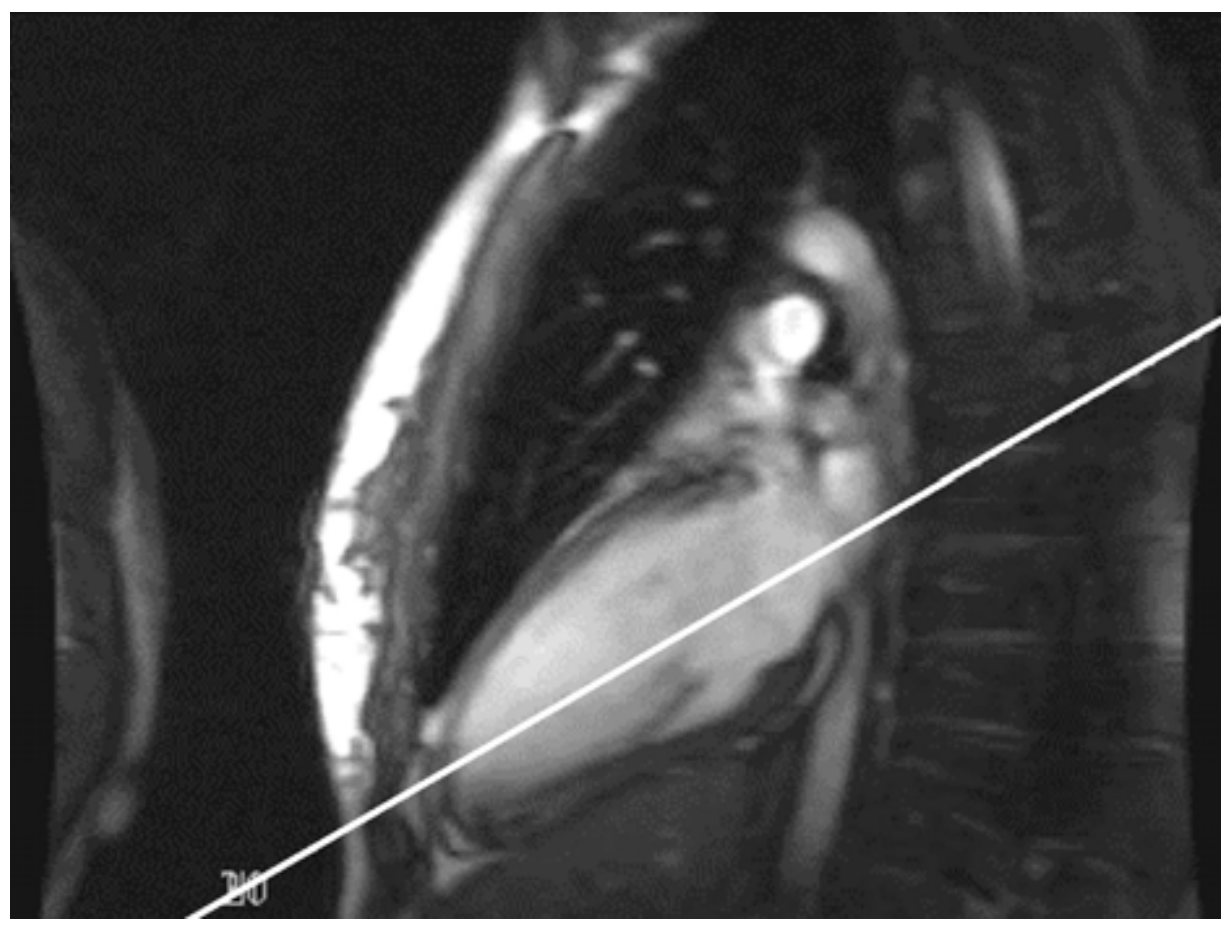

Figure A10.2.6 Prescription of a four-chamber view from the vertical long axis image.

Congenital Heart Disease

A10.2.9

Supplement 10 
Table A10.2.6 Primary Clinical Imaging Parameters for Sequence 5: Four-Chamber Cine Scout

Patient position

Scan type

Imaging plane (orientation)

Pulse sequence database (PSD)

Central slice or volume center

Echo time $\left(T_{\mathrm{E}}\right)$

Receiver bandwidth (RBW)

Number of lines per segment

Repeat time $\left(T_{\mathrm{R}}\right)$

Delay time $\left(T_{\mathrm{D}}\right)$

Flip angle (FA)

Fields of view $\left(\mathrm{FOV}_{x}, \mathrm{FOV}_{y}\right)$

Resolution $(\Delta x, \Delta y)$

Number of data points collected $\left(N_{\mathrm{x}}, N_{\mathrm{y}}\right)$

Display matrix $\left(D_{\mathrm{x}}, D_{\mathrm{y}}\right)$

Slice thickness $(\Delta z)$

Number of slices

Slice gap

Number of acquisitions $\left(N_{\text {acq }}\right)$

Swap read and phase encoding

Number of cardiac phases

ECG gating

Scan time
Supine

Cardiac cine gradient echo

Oblique coronal

2-D FIESTA/true-FISP/balanced FFE

Center of left ventricle

Minimum (e.g., $1.3 \mathrm{msec}$ )

$\pm 125 \mathrm{kHz}$

$12-16$

30-40 msec (temporal resolution)

Minimum (e.g., $10 \mathrm{msec}$ )

$45^{\circ}$

$360 \mathrm{~mm}, 360 \mathrm{~mm}$

$1.41 \mathrm{~mm}, 2.25 \mathrm{~mm}$

256,160

256,256

$8 \mathrm{~mm}$

1

NA

1

No

20-30 (heart rate dependent)

Yes

8-11 sec (10-14 R-to-R intervals)
Assessment of Right Ventricular Dysplasia

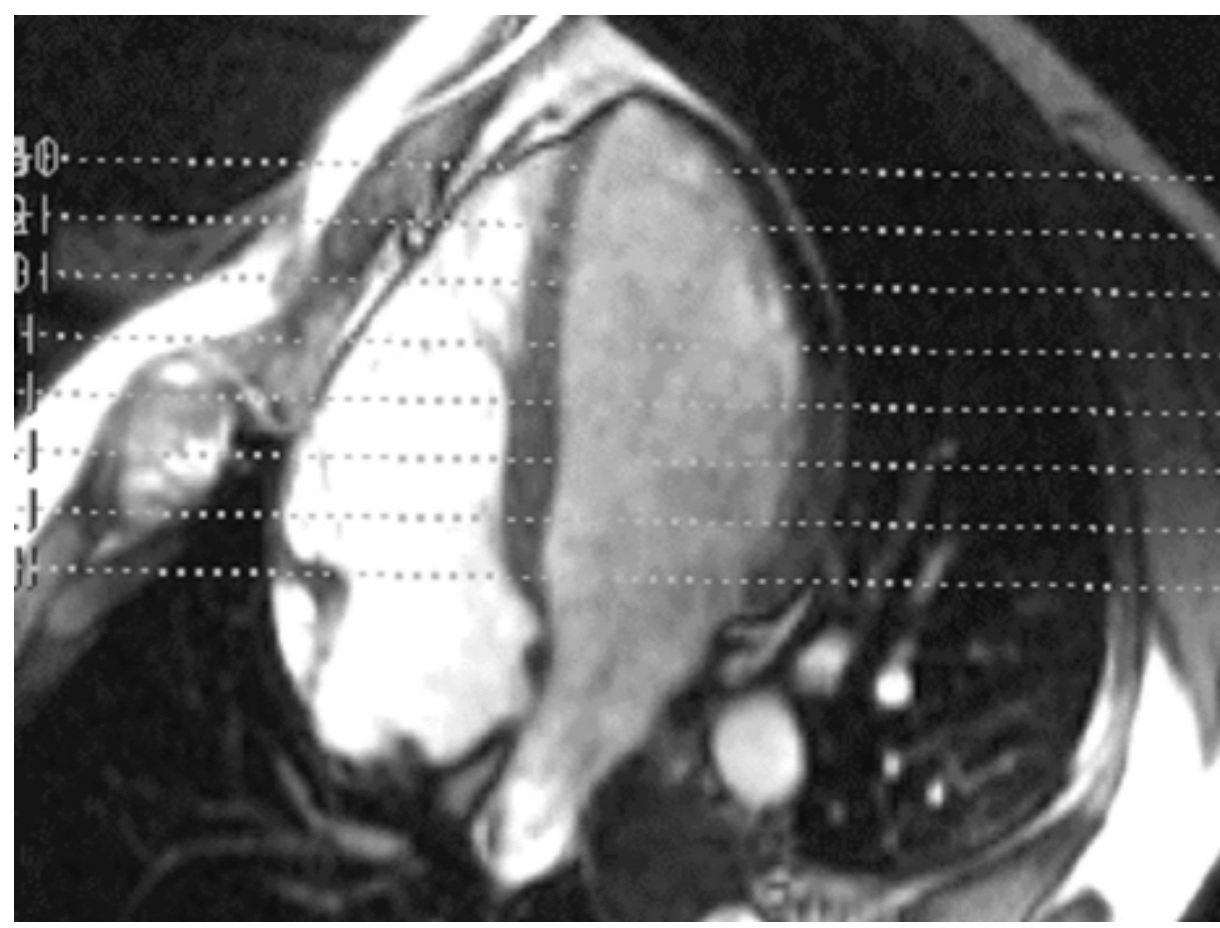

Figure A10.2.7 Prescription of short-axis cine sequence on the four-chamber diastolic image. Note that the coverage starts from the mitral valve plane to the apex of the ventricle. 
Table A10.2.7 Primary Clinical Imaging Parameters for Sequence 6: Short-Axis Cine

Patient position

Scan type

Imaging plane (orientation)

Pulse sequence database (PSD)

Central slice or volume center

Echo time $\left(T_{\mathrm{E}}\right)$

Receiver bandwidth (RBW)

Number of lines per segment

Repeat time $\left(T_{\mathrm{R}}\right)$

Delay time $\left(T_{\mathrm{D}}\right)$

Flip angle (FA)

Fields of view $\left(\mathrm{FOV}_{x}, \mathrm{FOV}_{y}\right)$

Resolution $(\Delta x, \Delta y)$

Number of data points collected $\left(N_{\mathrm{x}}, N_{\mathrm{y}}\right)$

Display matrix $\left(D_{\mathrm{x}}, D_{\mathrm{y}}\right)$

Slice thickness $(\Delta z)$

Number of slices

Slice gap

Number of acquisitions $\left(N_{\text {acq }}\right)$

Swap read and phase encoding

Slice locations

Number of cardiac phases

ECG gating

Scan time
Supine

Cardiac cine gradient echo

Oblique transverse

2-D FIESTA/true-FISP/balanced FFE

Center of heart

Minimum (e.g., $1.3 \mathrm{msec}$ )

$\pm 125 \mathrm{kHz}$

$12-16$

-30-40 msec (temporal resolution)

Minimum (e.g., $10 \mathrm{msec}$ )

$45^{\circ}$

$360 \mathrm{~mm}, 360 \mathrm{~mm}$

$1.41 \mathrm{~mm}, 2.25 \mathrm{~mm}$

256,160

256,256

$8 \mathrm{~mm}$

Variable (usually 6-8 in total)

$4 \mathrm{~mm}$

1

No

From the mitral plane to the apex of the heart

20-30 (heart rate dependent)

Yes

1-2 min (10-14 R-to-R intervals per slice)

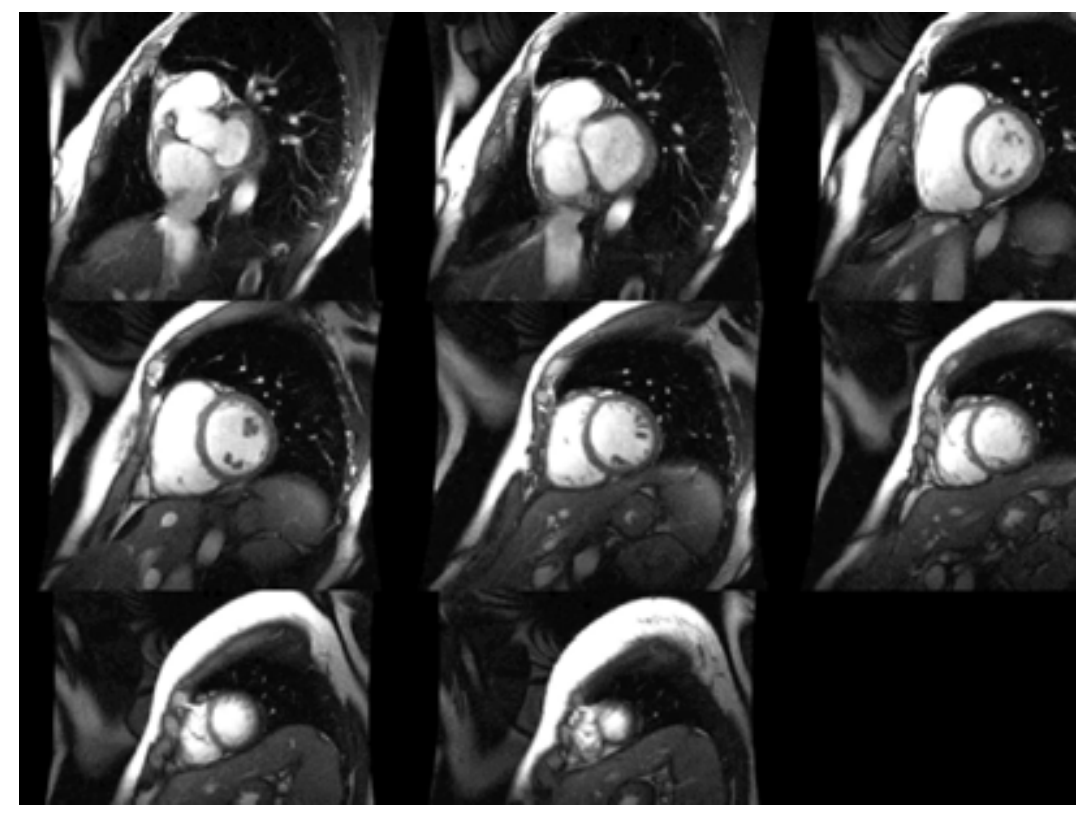

Figure A10.2.8 An example of short-axis bright blood cine images. Note that the coverage starts from the mitral valve plane to the apex of the left ventricle.

Congenital Heart Disease

A10.2.11 
Table A10.2.8 Primary Clinical Imaging Parameters for Sequence 7:

Short-Axis Black Blood Images

\begin{tabular}{|c|c|}
\hline Patient position & Supine \\
\hline Scan type & $\begin{array}{l}\text { Double inversion recovery fast-spin } \\
\text { echo }\end{array}$ \\
\hline Imaging plane (orientation) & Oblique transverse \\
\hline Pulse sequence database (PSD) & FSE-xl \\
\hline Central slice or volume center & Center of heart \\
\hline Echo time $\left(T_{\mathrm{E}}\right)$ & Minimum-full (e.g., 5-10 msec) \\
\hline Receiver bandwidth (RBW) & $\pm 62.5 \mathrm{kHz}$ \\
\hline Echo train length (ETL) & 24 (preferred) to 32 \\
\hline Repeat time $\left(T_{\mathrm{R}}\right)$ & 1 or $2 \mathrm{R}$-to-R intervals \\
\hline Inversion time $\left(T_{\mathrm{I}}\right)$ & $\begin{array}{l}T_{\mathrm{I}} \text { set by system (e.g., for } T_{\mathrm{R}} \text { of } 2 \\
\text { R-to-R intervals, } T_{\mathrm{I}} \text { is } 600 \mathrm{msec} \text {; for } 1 \\
\text { R-to-R interval, } T_{\mathrm{I}} \text { is } 200 \mathrm{msec} \text { ) }\end{array}$ \\
\hline Flip angle (FA) & $\mathrm{NA}^{a}$ \\
\hline Fields of view $\left(\mathrm{FOV}_{x}, \mathrm{FOV}_{y}\right)$ & $\begin{array}{l}260 \mathrm{~mm}, 260 \mathrm{~mm} \text { (depends on patient } \\
\text { size and available signal) }\end{array}$ \\
\hline Resolution $(\Delta x, \Delta y)$ & $1.02 \mathrm{~mm}, 1.02 \mathrm{~mm}$ \\
\hline Number of data points collected $\left(N_{\mathrm{x}}, N_{\mathrm{y}}\right)$ & 256,256 \\
\hline Display matrix $\left(D_{\mathrm{x}}, D_{\mathrm{y}}\right)$ & 256,256 \\
\hline Slice thickness $(\Delta z)$ & $5 \mathrm{~mm}$ \\
\hline Number of slices & 5 (total) \\
\hline Slice gap & $5 \mathrm{~mm}$ \\
\hline Number of acquisitions $\left(N_{\text {acq }}\right)$ & 1 \\
\hline Swap read and phase encoding & No \\
\hline Slice locations & $\begin{array}{l}\text { From the mitral plane to the apex of } \\
\text { the heart }\end{array}$ \\
\hline ZIP 512 & Yes \\
\hline Saturation pulses & $\begin{array}{l}\text { Anterior saturation band over } \\
\text { subcutaneous fat }\end{array}$ \\
\hline ECG gating & Yes \\
\hline Scan time & $\begin{array}{l}\sim 1 \mathrm{~min}\left(13-18 \mathrm{sec} \text { per slice when } T_{\mathrm{R}}=\right. \\
2 \mathrm{R} \text {-to- } \mathrm{R} \text { interval }) ; \sim 40 \mathrm{sec}(6-9 \mathrm{sec} \\
\left.\text { per slice when } T_{\mathrm{R}}=1 \mathrm{R} \text {-to- } \mathrm{R} \text { interval }\right)\end{array}$ \\
\hline
\end{tabular}

${ }^{a}$ The flip angle of the inversion pulse is $180^{\circ}$.

ventricular apex to the mid mitral valve plane as shown in Figure A10.2.6. Set up parameters of sequence 5 as shown in Table A10.2.6. Instruct the patient to take in a deep breath and exhale, repeat, take in another deep breath, and hold it. Run sequence 5.

Other than the scan plane, slice location, and number of slices, the parameters are identical to sequence 3.

On the long axis scout image prescribe four-chamber scout cine image as shown in Figure A10.2.6. This view provides a four-chamber view of the heart, similar to the echocardiographic four-chamber view. In this view one can assess the relative chamber sizes of the left and right ventricle and ventricular function visually.

\section{Sequence 6: Short-axis cine}

23. On the diastolic four-chamber scout image obtained from sequence 5, place the localizer line parallel to the mitral valve plane and perpendicular to the long axis of the left ventricle. Draw the grid lines at the mitral valve plane and extend them up to the left ventricular apex as shown in Figure A10.2.7. Set up parameters as shown in Table A10.2.7 with number of slices set to one. 


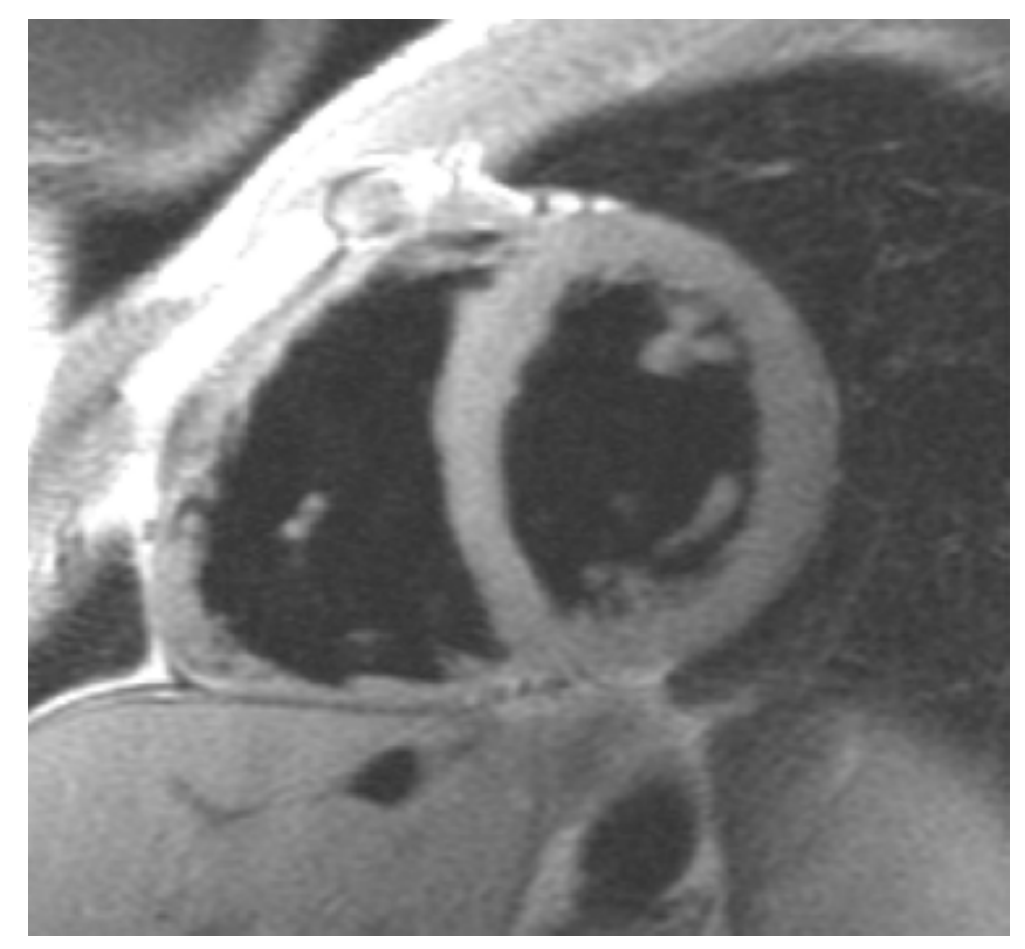

Figure A10.2.9 A short-axis black blood image.

24. Instruct the patient to take in a deep breath and exhale, repeat, take in another deep breath, and hold it. Run sequence 6 according to Table A10.2.7.

25. Repeat step 24 until the entire region of interest is covered.

The parameters in Table A10.2.7 are similar to those in Table A10.2.4. An example of short axis cine images is shown in Figure A10.2.8. Coverage for this sequence should start from the mitral valve plane to the apex of the heart. This is important as this view is often used for quantification of ventricular volumes. Manual prescan should be performed in GE scanners to optimize image quality.

\section{Sequence 7: Short-axis black blood images}

26. Instruct the patient to take in a deep breath and exhale, repeat, take in another deep breath, and hold it. Run sequence 7 according to Table A10.2.8 with number of slices set to one.

27. Repeat step 26 until the entire region of interest is covered.

The parameters for this sequence are similar to those used in sequence 2.

An example of good quality short axis black blood images is shown in Figure A10.2.9.

This view is useful to assess the inferior (diaphragmatic) border of the right ventricle. Care should be taken to adjust the field of view appropriately to minimize wrap artifacts. In scanners with a surface coil, using anterior coil elements only and switching off the posterior coil to reduce wrap artifacts is recommended.

\section{Sequence 8: Transverse black blood images with fat suppression (optional)}

28. Set up imaging parameters for this sequence as shown in Table A10.2.9. Copy the prescription from sequence 2 on to this sequence. Make sure the option of fat suppression is chosen. Manually align the imaging center frequency to water peak, while maximizing fat suppression. 
Table A10.2.9 Primary Clinical Imaging Parameters for Sequence 8: Transverse Black Blood Images with Fat Suppression

Patient position

Scan type

Imaging plane (orientation)

Pulse sequence database (PSD)

Central slice or volume center

Echo time $\left(T_{\mathrm{E}}\right)$

Receiver bandwidth (RBW)

Echo train length (ETL)

Repeat time $\left(T_{\mathrm{R}}\right)$

Inversion time $\left(T_{\mathrm{I}}\right)$

Flip angle (FA)

Fields of view $\left(\mathrm{FOV}_{x}, \mathrm{FOV}_{y}\right)$

Resolution $(\Delta x, \Delta y)$

Number of data points collected $\left(N_{\mathrm{x}}, N_{\mathrm{y}}\right)$

Display matrix $\left(D_{\mathrm{x}}, D_{\mathrm{y}}\right)$

Slice thickness $(\Delta z)$

Number of slices

Slice gap

Number of acquisitions $\left(N_{\text {acq }}\right)$

Swap read and phase encoding

Slice locations

ZIP 512

Saturation pulses

Fat suppression

ECG gating

Scan time
Supine

Double inversion recovery fast-spin

echo

Transverse

FSE-xl

Center of heart

Minimum-full (e.g., $3.1 \mathrm{msec}$ )

$\pm 62.5 \mathrm{kHz}$

24 (preferred) to 32

2 R-to-R intervals

$\sim 625 \mathrm{msec}$

$\mathrm{NA}^{a}$

$260 \mathrm{~mm}, 260 \mathrm{~mm}$ (depends on patient size and available signal)

$1.02 \mathrm{~mm}, 1.02 \mathrm{~mm}$

256,256

256,256

$5 \mathrm{~mm}$

Variable (usually 8-9 in total)

$5 \mathrm{~mm}$

1

No

From pulmonary bifurcation to dome of diaphragm

Yes

Chemical shift fat suppression

Yes

Yes

2-3 min (13-18 sec per slice)

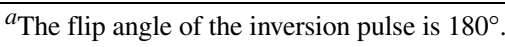

29. Instruct the patient to take in a deep breath and exhale, repeat, take in another deep breath, and hold it. Run sequence 8 according to Table A10.2.9 with number of slices set to one.

30. Repeat step 29 until the entire region of interest is covered.

The prescription for this sequence is exactly the same as in sequence 2.

An example of good quality transverse black blood fat suppressed image is shown in Figure A10.2.10.

This sequence is optional and usually requires an additional 10 min to the total imaging time. The authors recommend performing this sequence if time permits in all patients. This sequence is particularly useful in patients who have abundant epicardial fat, which makes it difficult to distinguish the true myocardial border. Also in patients who have had sub-optimal image quality in the non-fat-suppressed transverse black blood images, repeating the sequence with fat suppression may improve confidence in diagnosis.

Fat-suppressed transverse black blood images are acquired at the authors' institution using double inversion recovery fast-spin echo sequence. If the center has a dedicated cardiac coil, best results are obtained using the anterior coil elements only (posterior coil switched off) to prevent wrap around artifacts. Also, the authors recommend using an anterior saturation band as shown in Figure A10.2.2. 


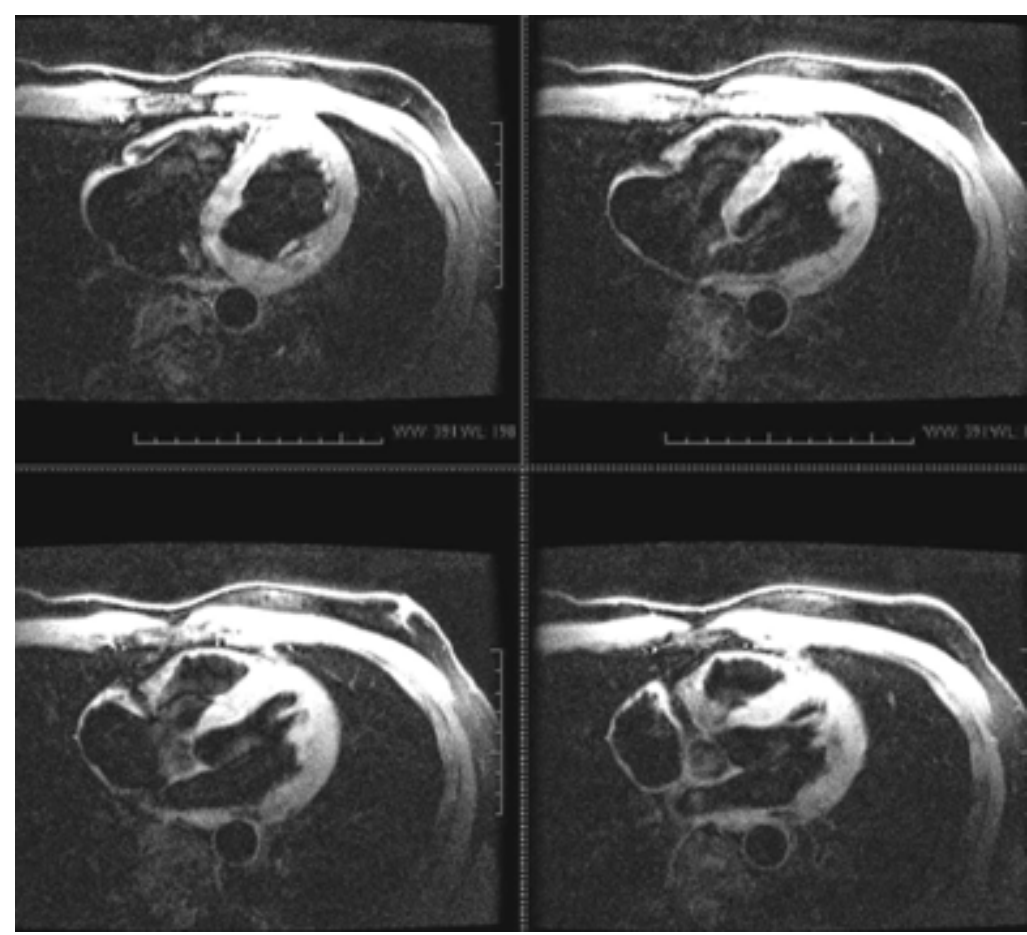

Figure A10.2.10 Transverse black blood images with fat suppression. Note the use of anterior coil elements only.

\section{COMMENTARY}

\section{Background Information}

Arrhythmogenic right ventricular dysplasia (ARVD) is a heritable cardiomyopathy characterized by fibro-fatty infiltration of the right ventricular (RV) myocardium (Marcus et al., 1982). Affected individuals have increased incidence of sudden death due to malignant ventricular arrhythmias. The disease is more common in men and is a substantial cause of sudden deaths in athletes $<35$ years old (Thiene et al., 1988). Fibro-fatty infiltration of the RV leads to progressive RV failure and, in the late stages of the disease process, the left ventricle may also be involved (Marcus et al., 1982; Thiene et al., 1988). The diagnosis of ARVD is based on a set of major and minor criteria encompassing structural, electrocardiographic and histological criteria proposed by the Task Force of the Working Group on Cardiomyopathies in 1994 (McKenna et al., 1994). As such, the accurate evaluation of right ventricular anatomy and function is crucial to diagnosis of this disease.

Conventional non-invasive imaging modalities like the echocardiogram and radionuclide ventriculography have several limitations, especially in patients with abnormal RV geome- try. By contrast, magnetic resonance (MR) imaging provides excellent anatomic details of the $\mathrm{RV}$ with high spatial and temporal resolutions. Also, it alone has the ability to depict tissue characterization non-invasively. For the above reasons, MR imaging is considered the non-invasive imaging modality of choice to evaluate ARVD (Pennell and Casolo, 1997). The improved contrast between the blood pool and the myocardium allows accurate and highly reproducible quantitation to be performed making it ideal for follow-up evaluation and to study the disease progression.

\section{Critical Parameters and Troubleshooting}

\section{Cardiac gating}

An adequate ECG tracing is crucial for cardiovascular MR imaging. For best results the authors recommend the use of the NuPrep gel to clean the skin prior to the application of the MR-compatible ECG leads. Excess hair needs to be shaved in order to ensure adequate skin contact. The use of fiber-optic leads decreases patient motion artifacts and yields better results.
Congenital Heart Disease

A10.2.15 


\section{Cardiac arrhythmias}

A significant proportion of patients being evaluated for ARVD have frequent ventricular ectopy, which can be problematic. The following is recommended to improve image quality in these patients.

Ideally, if the patient is known to have frequent ectopy, a beta blocker $(50 \mathrm{mg}$ Metoprolol) given orally 1 to $2 \mathrm{hr}$ prior to the scan minimizes ectopy. Often this information becomes apparent while the patient is on the scanner table. If the ectopy are frequent, then the resulting scan quality will be poor and the test becomes uninterpretable. In this case, it is prudent to reschedule the scan at a later time on the same day if the schedule permits and to give the patient a beta blocker while waiting. If it is not possible to reschedule the scan on the same day, then do not perform the scan and reschedule it for another day with adequate beta blocker pre-medication.

\section{Breathing artifacts}

All images should be obtained during the breath-hold to minimize motion artifacts. Adequate patient education to make sure that the patient understands the importance of breath holding is invaluable. The patient should be told that movement and/or speaking during the exam will cause the images to be less than optimal, and if at all possible to refrain from such activity during the scan. For pediatric patients performing exams without breath holding, shallow breathing is recommended. In spite of all this, a proportion of patients fail to hold their breath. In pediatric patients, an alternative is to insure the number of signal averages, e.g., to 4, and perform the exam without breath-holding. Inversion prepared HASTE sequences are an alternative. In both cases, the diagnostic value of black blood images is substantially reduced. The use of an anterior saturation band over the subcutaneous fat of the anterior chest wall will help reduce breathingrelated motion artifacts.

\section{Anticipated Results}

The diagnosis of ARVD is made in the presence of major and minor criteria proposed by the Task Force of cardiomyopathies (McKenna et al., 1994). These criteria encompass electrical and morphologic abnormalities of the right ventricle. The goal of MR imaging in ARVD is to accurately depict RV structure and function. MR abnormalities that are currently included in the Task Force criteria are RV chamber enlargement and RV global and/or regional dys- function. Intramyocardial fat on MR imaging is currently not recognized as a criteria for ARVD by the Task Force, as the reliability of this finding is unknown. When evaluating RV structure, special attention should be paid to the $\mathrm{RV}$ inflow, RV apex, and the outflow tract, as these are the commonly involved sites in ARVD. Fat infiltration can often be difficult to visualize, as epicardial fat is normally present abundantly near the atrio-ventricular groove and near the RV apex. A clear line of demarcation often exists between the epicardial fat and the RV myocardium in normal subjects and a disruption of this line is often seen in patients with ARVD. There is also increased trabeculation of the RV with a prominent moderator band in patients with ARVD. Assessing RV function visually can be very challenging as the RV has a complex morphology and the contraction pattern differs from the left ventricle. The authors recommend assessing RV function from the transverse bright blood cine images. The RV on transverse cine images starts off as a large triangle in diastole, which becomes a smaller triangle in systole. Most of the contraction occurs in the long-axis of the RV from the movement of the tricuspid valve towards the RV apex. The RV anterior wall should be examined for bulges and aneurysms, as these are morphologic abnormalities associated with ARVD. Whenever feasible, quantification of RV volumes and ejection fraction should be performed. Quantification of RV volumes can be performed on the short-axis bright blood images using MASS software (Medis). Endocardial margins of the end diastolic and end systolic images are manually contoured and volumes calculated using the "Simpson's" rule. These are more reproducible than qualitative estimates and may increase sensitivity of MR imaging.

\section{Acknowledgements}

This work was supported in part by the Johns Hopkins ARVD center (funded by a private grant from the Bogle Foundation) and National Institutes of Health Research Grant 1 UO1 HL65594-01 A1 (D. Bluemke, PI, MRI ARVD reading center). The authors would like to thank Scott Pride, R.T., and Donna Green, R.T. for providing helpful comments.

\section{Literature Cited}

Marcus, F.I., Fontaine, G.H., Guiraudon, G., Frank, R., Laurenceau, J.L., Malergue, C., and Grosgogeat, Y. 1982. Right ventricular dysplasia: A report of 24 adult cases. Circulation 65:384398.
Assessment of Right Ventricular Dysplasia

A10.2.16 
McKenna, W.J., Thiene, G., Nava, A., Fontaliran, F., Blomstrom-Lundqvist, C., Fontaine, G., and Camerini, F. 1994. Diagnosis of arrhythmogenic right ventricular dysplasia/cardiomyopathy. $\mathrm{Br}$. Heart J. 71:215-218.

Pennell, D. and Casolo, G. 1997. Right ventricular arrhythmia: Emergence of magnetic resonance imaging as an investigative tool. Eur. Heart J. 18:1843-1845.

Thiene, G., Nava, A., Corrado, D., Rossi, L., and Pennelli, N. 1988. Right ventricular cardiomyopathy and sudden death in young people. N. Engl. J. Med. 318:129-133.
Internet Resources

http://www.arvd.com

An Internet site providing an overview of clinical features of ARVD along with a current MRI protocol at the authors' institution.

Contributed by Harikrishna Tandri and David A. Bluemke

The Johns Hopkins University School of Medicine

Baltimore, Maryland
Congenital Heart

Disease

A10.2.17 